\title{
Search for superresolution in a metamaterial solid immersion lens
}

Novitsky, Andrey; Repän, Taavi; Malureanu, Radu; Takayama, Osamu; Shkondin, Evgeniy; Laurynenka, Andrei

Published in:

Physical Review A

Link to article, DOI:

10.1103/PhysRevA.99.023835

Publication date:

2019

Document Version

Publisher's PDF, also known as Version of record

Link back to DTU Orbit

Citation (APA):

Novitsky, A., Repän, T., Malureanu, R., Takayama, O., Shkondin, E., \& Laurynenka, A. (2019). Search for superresolution in a metamaterial solid immersion lens. Physical Review A, 99(2), [023835].

https://doi.org/10.1103/PhysRevA.99.023835

\section{General rights}

Copyright and moral rights for the publications made accessible in the public portal are retained by the authors and/or other copyright owners and it is a condition of accessing publications that users recognise and abide by the legal requirements associated with these rights.

- Users may download and print one copy of any publication from the public portal for the purpose of private study or research.

- You may not further distribute the material or use it for any profit-making activity or commercial gain

- You may freely distribute the URL identifying the publication in the public portal

If you believe that this document breaches copyright please contact us providing details, and we will remove access to the work immediately and investigate your claim 


\title{
Search for superresolution in a metamaterial solid immersion lens
}

\author{
A. Novitsky, ${ }^{1,2}$ T. Repän, ${ }^{1}$ R. Malureanu, ${ }^{1}$ O. Takayama, ${ }^{1}$ E. Shkondin,,${ }^{1}$ and A. V. Lavrinenko ${ }^{1}$ \\ ${ }^{1}$ DTU Fotonik, Technical University of Denmark, Ørsteds Plads 343, DK-2800 Kongens Lyngby, Denmark \\ ${ }^{2}$ Department of Theoretical Physics and Astrophysics, Belarusian State University, Nezavisimosti Avenue 4, 220030 Minsk, Belarus
}

(Received 20 December 2018; published 19 February 2019)

\begin{abstract}
Superresolution is an important feature needed for modern optical microscopy. It can be achieved by using the transmission of evanescent waves in hyperbolic metamaterials. However, such devices suffer from material losses. Here we investigate a recently proposed metamaterial solid immersion lens-an assembly of dielectric nanoparticles. Using the multiple-scattering theory we reveal conversion of evanescent to propagating waves under conditions of coherent scattering. However, efficiency of the conversion is rather low as confirmed by the transmission of the fields of a couple of point sources. Comparing the scattering of light of a spherical cluster of nanospheres with that of a solid sphere of the same radius we find the same far fields in both cases. Generally, our conclusion is that material in the form of the assembly of nanoparticles behaves like an effective medium and does not demonstrate the superresolution in the far field. We believe that the resolution of the metamaterial solid immersion lens has the same origin as that of the conventional sold immersion lens.
\end{abstract}

DOI: 10.1103/PhysRevA.99.023835

\section{INTRODUCTION}

A lens has a notorious limitation of resolution power related to the damping of evanescent waves emitted by a source. Metamaterials as artificial media with subwavelength unit cells offer promising approaches for transmission of evanescent waves. An ideal superlens as a negative-refractiveindex slab is able to create a perfect image [1]. A hyperlens made of the material with an indefinite permittivity tensor (so-called hyperbolic metamaterial $[2,3]$ ) was shown to beat the diffraction limit [4]. A dielectric-type hyperbolic metamaterial can be used for fabrication of bright-field hyperlenses $[5,6]$, while the metallic-type one can be exploited for dark-field microscopy [7,8]. Metamaterial flat lenses [9,10] and metasurfaces [11-13] have been proposed for subwavelength imaging as well. However, realistic superlenses and hyperlenses suffer from the inevitable material losses degrading their performance due to the metallic inclusions. All-dielectric metamaterials are actively considered nowadays. Being able to support optical magnetism [14], they can substitute conventional metamaterials in various applications, including invisibility cloaking [15,16] and skin-depth engineering [17]. Peculiar scattering properties of individual high-refractiveindex nanoparticles based on the excitation of electric and magnetic multipole moments can be exploited for tuning the linear and nonlinear response from the metamaterials and metasurfaces [18-20].

Another tool to improve the imaging resolution is to apply a solid immersion lens (SIL) [21], a thick lens on the micro[22] and nanoscale [23] having both a higher magnification and numerical aperture due to filling of the object space with a high-refractive-index solid material. The resolving power of a conventional oil immersion microscope [24] (a planar slab of oil with no magnification) is $\delta=\lambda / 2 \mathrm{NA}$, where $\lambda$ is the wavelength, $\mathrm{NA}=n \sin \theta$ is the numerical aperture, $n$ is the oil refractive index, and $\theta$ is the maximal incident angle of the waves coming to an objective. When all waves are accepted by the objective $\left(\theta=90^{\circ}\right)$, the resolving power takes its maximum value $\delta=\lambda / 2 n$. Enhancement of the resolving power is explained by the transmission of both propagating $\left(\left|k_{t}\right| \leqslant k_{0}\right)$ and evanescent $\left(k_{0} \leqslant\left|k_{t}\right| \leqslant n k_{0}\right)$ waves incident from air [25], where $k_{t}$ is the tangential wave-vector component with respect to the slab and $k_{0}=2 \pi / \lambda$ is the wave number in vacuum. A SIL is also characterized by a magnification factor $M$. Having two input objects remote from each other at a distance of $d$, one observes the distance $M d$ between the objects at the output. This can be achieved by using a spherical shape of the SIL, e.g., a truncated spherical lens. Then in the reciprocal space the output transverse component of the wave vector decreases as $k_{t} / M$ enlarging the range of propagating waves defined by the following condition: longitudinal components of the wave vector $\left(k_{0}^{2} n^{2}-k_{t}^{2} / M^{2}\right)^{1 / 2}$ should be real-valued. The admitted transverse components are then $-n M k_{0} \leqslant k_{t} \leqslant n M k_{0}$, and the numerical aperture is NA $=n M$. Hence, the shortest resolved distance is $\delta=\lambda / 2 n M$. In general, the magnification factor of the SIL depends on its shape, e.g., a hemispherical SIL increases the numerical aperture by $M=n$.

Recently, a novel type of the SIL was proposed-a metamaterial solid immersion lens (mSIL) [26,27]. It is a cluster of dielectric nanospheres in the shape of a micron-sized truncated sphere fabricated by using the nano-solid-fluid assembly technique. The authors reported on the superresolution properties of the mSILs and explained them by using the conversion of evanescent waves into propagating waves. Such a conversion is accompanied with a guiding-like effect for nondiffractive wave propagation through a cluster of nanospheres revealed numerically. Here we revisit the aforementioned explanation by using the electric dipole (Rayleigh) approximation. We consider a finite assembly of nanospheres and examine it for the evanescent-wave and point-source field propagation. 

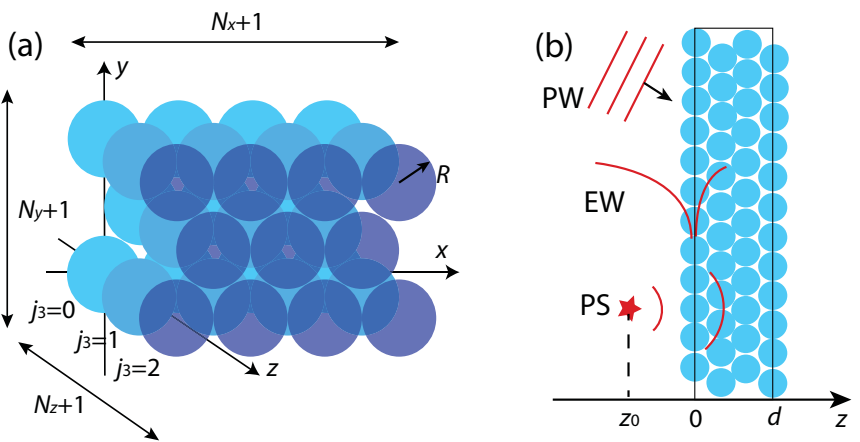

FIG. 1. Sketches of the (a) assembly of $\left(N_{x}+1\right) \times\left(N_{y}+1\right) \times$ $\left(N_{z}+1\right)$ nanospheres and (b) field propagation through the slab. In panel (a), different colors of the particles correspond to different coordinates $z=2 \sqrt{2 / 3} R j_{3}$. In panel (b), the incidence of the plane wave (PW), evanescent wave (EW), and field of the point source (PS) are schematically shown.

The article consists of six sections including the introduction and conclusion. In Sec. II, we discuss a calculation technique for electromagnetic wave propagation in a finite cluster of dipolar nanospheres. The technique is employed for analysis of the conversion of evanescent to propagating waves in Sec. III and propagation of point-source radiation in Sec. IV. In Sec. V, we calculate light scattering of a pointsource field by the solid sphere and cluster of nanospheres of the same radius making conclusion on their similarity in the far field.

\section{WAVE PROPAGATION IN A CLUSTER OF NANOSPHERES}

Wave propagation in mSILs can be described with the help of the multiple-scattering theory [28-30]. Here we will briefly discuss it for a certain case of electrically small closely packed nanoparticles. A stationary electromagnetic beam having electric field $\mathbf{E}_{\mathrm{inc}}(\mathbf{r})$ and wavelength $\lambda$ is incident on a densely packed assembly of equivalent nanospheres (radius $R$ and refractive index $n$ ) in air. We deal with an AB close packing defined as every second layer of nanospheres is the same, that is the sequence of layers can be written ABABAB...[Fig. 1(a)]. We write the positions of the nanosphere centers by using the radius vector (see Appendix A for details)

$$
\mathbf{r}_{j_{1}, j_{2}, j_{3}}=j_{1} \mathbf{b}_{1}+\left[\operatorname{Mod}\left(3 j_{1}-2 j_{3}, 6\right)-6 j_{2}\right] \mathbf{b}_{2}+j_{3} \mathbf{b}_{3},
$$

where $\mathbf{b}_{1}=R \mathbf{e}_{x}, \mathbf{b}_{2}=\frac{R}{\sqrt{3}} \mathbf{e}_{y}, \mathbf{b}_{3}=\frac{2 \sqrt{2} R}{\sqrt{3}} \mathbf{e}_{z}$, while $\operatorname{Mod}\left(3 j_{1}-\right.$ $\left.2 j_{3}, 6\right)$ means the remainder of the division by six. If the indices along the Cartesian axes [see Fig. 1(a)] span the values $-N_{x} / 2 \leqslant j_{1} \leqslant N_{x} / 2,-N_{y} / 2 \leqslant j_{2} \leqslant N_{y} / 2$, and $0 \leqslant j_{3} \leqslant N_{z}$, then the total number of spheres in the cluster equals $\left(N_{x}+\right.$ 1) $\left(N_{y}+1\right)\left(N_{z}+1\right)$.

Supposing that the radii of the nanospheres are much smaller than the radiation wavelength $\lambda \gg R$, the nanospheres can be accurately modeled as electric dipoles with moments equal to $\mathbf{p}=\alpha \mathbf{E}$. Here $\alpha$ is the sphere polarizability and $\mathbf{E}$ is the external electric field at the center of the sphere. The external field is a superposition of the incident field and that radiated by other dipoles in the assembly:

$$
\begin{aligned}
\mathbf{p}_{j_{1}, j_{2}, j_{3}}= & \alpha\left[\mathbf{E}_{\mathrm{inc}}\left(\mathbf{r}_{j_{1}, j_{2}, j_{3}}\right)\right. \\
& \left.+\sum_{j_{1}^{\prime}, j_{2}^{\prime}, j_{3}^{\prime}} \hat{G}_{E}\left(\mathbf{r}_{j_{1}^{\prime}, j_{2}^{\prime}, j_{3}^{\prime}}-\mathbf{r}_{j_{1}, j_{2}, j_{3}}\right) \mathbf{p}_{j_{1}^{\prime}, j_{2}^{\prime}, j_{3}^{\prime}}\right],
\end{aligned}
$$

where the electric Green's function is

$\hat{G}_{E}(\mathbf{r})=\frac{k_{0}^{2}}{r^{3}} e^{i k_{0} r}\left[r^{2}-\mathbf{r} \otimes \mathbf{r}+\frac{i}{k_{0} r}\left(1+\frac{i}{k_{0} r}\right)\left(r^{2}-3 \mathbf{r} \otimes \mathbf{r}\right)\right]$

for $\mathbf{r} \neq 0$ and $\hat{G}_{E}(0)=0$. It is used for writing electric fields $\hat{G}_{E} \mathbf{p}$ of electric dipoles. Here $\mathbf{a} \otimes \mathbf{b}$ is an outer (tensor) product of vectors $\mathbf{a}$ and $\mathbf{b}$.

We introduce two methods of calculations. If Eq. (2) is solved without further assumptions, it is a direct calculation (see Appendix B). In general, this approach is demanding for numerical resources. That is why we simplify it by reducing the number of dimensions to two or even one. Twodimensional (2D) calculations are approximately valid, when the incident field depends on two coordinates as $\mathbf{E}_{\mathrm{inc}}(x, z)$, while the number of nanospheres in the $y$ direction is great. Validity of one-dimensional (1D) calculations is justified for the fields $\mathbf{E}_{\text {inc }}(z)$ and as great as possible a number of nanospheres in the plane $(x, y)$. Another method exploits the lattice-sum approximation for finite structures (see Appendix C). As well as 1D direct calculations, it is valid for a large number of nanoparticles in the plane $(x, y)$. The details of calculation techniques and their comparison can be found in Appendix D.

Throughout the paper we employ a number of default values for calculations taken from Ref. [26]: the nanosphere's radius $R=7.5 \mathrm{~nm}$ and refractive index $n=2.55$ (anatase $\mathrm{TiO}_{2}$ ), the wavelength of the incident wave $\lambda=470 \mathrm{~nm}$, and the effective refractive index of the assembly $n_{\text {eff }}=2$. The assembly of nanospheres is assumed to be free standing in air. Since $R \ll \lambda\left(k_{0} R=2 \pi R / \lambda \approx 0.1\right)$, the nanospheres can be treated as electric dipoles, and the theory outlined above is applicable. The electric-dipole moment of the nanospheres is written as $\mathbf{p}=\alpha \mathbf{E}$ with electric polarizability $\alpha=\alpha_{0} /(1-$ $\left.2 i k_{0}^{3} \alpha_{0} / 3\right)$, where $\alpha_{0}=R^{3}\left(n^{2}-1\right) /\left(n^{2}+2\right)$ is a static electric polarizability. Neglecting the radiative-reaction correction term $2 i k_{0}^{3} \alpha_{0} / 3$ within the approximation $k_{0} R \ll 1$, we further adopt $\alpha=\alpha_{0}$.

\section{PROPAGATION OF EVANESCENT WAVES}

To judge the resolution improvement of metamaterial solid immersion lenses we need to know the properties of an evanescent wave transmission. The incident field of an evanescent wave (transverse wave number $k_{x}>k_{0}$ ) reads

$$
\mathbf{E}_{\text {inc }}=E_{0} \exp \left(i k_{x} x-\sqrt{k_{x}^{2}-k_{0}^{2}} z\right) \mathbf{e}_{y},
$$

where $E_{0}$ is the field amplitude. For the field $\mathbf{E}_{\text {inc }}(x, z)$ the $2 \mathrm{D}$ direct calculation technique is applicable.

According to Ref. [26] conversion of incident evanescent waves into propagating ones, their guiding and further emission is a mechanism behind the superresolution of the 

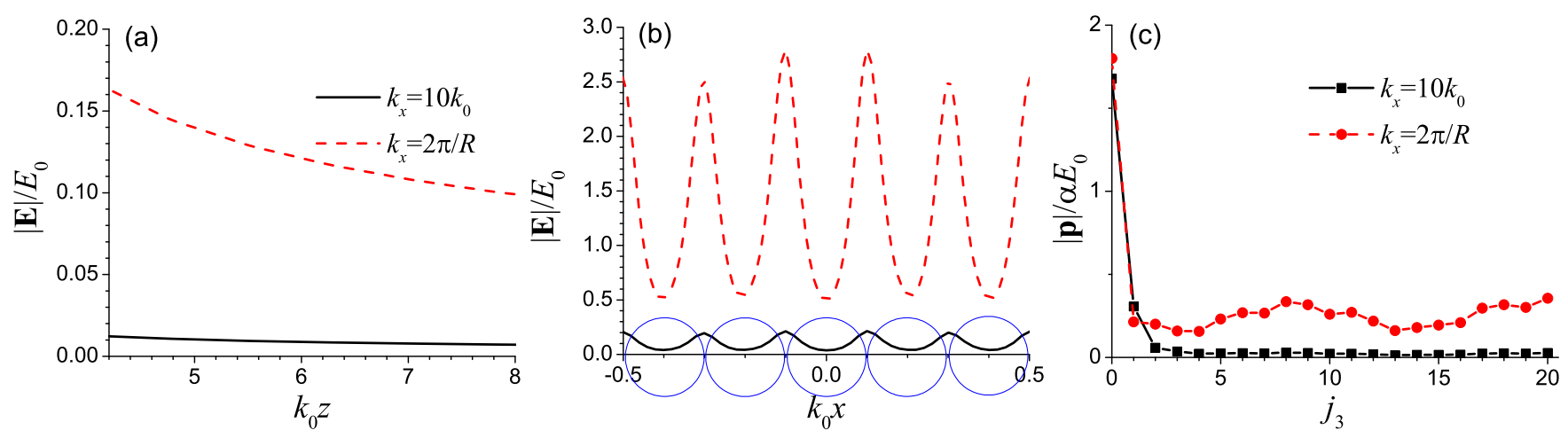

FIG. 2. 2D direct calculation of the evanescent wave propagation for $k_{x}=10 k_{0}$ and $k_{x}=2 \pi / R$. (a) Transmission coefficient $|\mathbf{E}| / E_{0}$ of the slab of nanospheres as a function of dimensionless coordinate $k_{0} z(x=y=0)$. (b) Transmission coefficient $|\mathbf{E}| / E_{0}$ as a function of $k_{0} x$ at $z=2 \sqrt{2 / 3} R N_{z}$ corresponding to the center of the last sphere in the slab (circles demonstrate positions of the nanospheres). (c) Dimensionless electric-dipole moments $|\mathbf{p}| / \alpha E_{0}$ of the nanospheres vs $j_{3}$ (coordinate $z$ ) at $j_{1}=j_{2}=0$. Geometrical parameters of the slab: $N_{x}=20, N_{y}=80$, and $N_{z}=20$.

mSIL. This effect can be tested for a planar slab composed of dielectric nanospheres shown in Fig. 1(b). In Fig. 2 we demonstrate a transmission of the evanescent waves. The values of the transmitted evanescent fields are much less than those of the normally incident waves shown in Fig. 8. The transverse distribution of the transmitted electric field in Fig. 2(b) agrees well with that demonstrated in Ref. [26]. The intrinsic structure of the periodically arranged assembly exhibits itself by means of the nonmonotonic dependence of the transmitted fields on the wave number $k_{x}$. For a homogeneous dielectric slab one expects the decrease of the transmission, when $k_{x}$ increases because of the factor $\exp \left[-\left(k_{x}^{2}-k_{0}^{2}\right)^{1 / 2} z\right]$. However, a periodic structure has the distinctive feature related to coherent excitation. The latter is available, when the wave number $k_{x}$ is a multiple of the reciprocal-lattice vector $2 \pi / R$ (the nanospheres are spaced with distance $R$ in the $x$ direction). The coherent excitation means that all electric dipoles oscillate in phase, thus substantially increasing the transmission. In Fig. 2 we observe that, in spite of $2 \pi / R \approx$ $62.7 k_{0}>10 k_{0}$, the coherent evanescent wave has much higher transmission than the low- $k_{x}$ evanescent wave. From Fig. 2(c) we also notice that the electric-dipole moment is oscillating with coordinate $z$, which is the attribute of a propagating field (such an oscillating behavior was also noticed in Ref. [26]). Thus, the coherent evanescent waves are indeed converted to propagating waves in the slab and, hence, are emitted as propagating waves as well. It is worth noticing that, if the excitation is in antiphase $\left(k_{x}=m \pi / R\right.$, where $m$ is an integer number), the destructive interference quenches the output field.

In the continuous-medium immersion lenses, the evanescent waves are converted to propagating waves enhancing the transmission, if the transverse wave-vector components are in the range $-n_{\text {eff }} k_{0}<k_{x}<n_{\text {eff }} k_{0}$. In Fig. 2 we observe negligible dipole moments and electric fields outside this range, for $k_{x}=10 k_{0}$. When the incident evanescent wave coherently excites the nanospheres, the dispersion equation

$$
\left(k_{x}-\frac{2 \pi m}{R}\right)^{2}+k_{z}^{2}=\frac{n_{\mathrm{eff}}^{2} \omega^{2}}{c^{2}}
$$

allows a number of bands, for which $k_{x}$ satisfies

$$
\frac{2 \pi m}{R}-\frac{n_{\mathrm{eff}} \omega}{c}<k_{x}<\frac{2 \pi m}{R}+\frac{n_{\mathrm{eff}} \omega}{c} .
$$

Conventional propagating waves are within the band $m=$ 0 . For the adopted parameters of the system, inequality (6) reads $62.7 m-2<k_{x} / k_{0}<62.7 m+2$. It is important that some evanescent waves are indeed converted into propagating waves, but not all evanescent waves, as was suggested in Ref. [26].

According to Eq. (6) the allowed bands can be expanded for larger refractive indexes of the nanospheres, i.e., for larger effective refractive indexes. For the larger nanosphere radius $R$ the allowed bands occupy most of the reciprocal space; that is, the overall transmission is expected to grow. Contrariwise, when $R \rightarrow 0$ (the limit of a continuous medium), only extremely-high $-k_{x}$ evanescent waves are allowed to propagate, but their contribution is normally negligible. All allowed bands merge, when $\frac{2 \pi}{R}=2 n_{\text {eff }} \omega / c$, that is $R=\lambda / 2 n_{\text {eff }}$. However, in this case the nanosphere size parameter $k_{0} R=\pi / n_{\text {eff }} \sim 1$ and, therefore, we go beyond the electric-dipole (Rayleigh) approximation: magnetic and electric-dipole moments should be taken into account and the above analysis ceases to be valid. Thus, we may claim that the Rayleigh nanospheres of the radius as large as possible should be chosen to increase efficiency of the excitation of the electric-dipole moments by evanescent waves and, therefore, the wave propagation to the far field.

Furthermore, we propose an explanation of the conversion of evanescent to propagating waves. An incident evanescent wave carries its energy in the $x$ direction and its energy flux in the cluster reads

$$
\Phi_{x}^{e v}=\int_{0}^{(2 \kappa)^{-1}} \frac{c L_{y}}{8 \pi} \operatorname{Re}\left(\mathbf{E} \times \mathbf{H}^{*}\right)_{x} d z=\frac{c L_{y} k_{x}\left(1-e^{-1}\right)}{16 \pi k_{0} \kappa},
$$

where $\kappa=\left(k_{x}^{2}-k_{0}^{2}\right)^{1 / 2}$ and $L_{y}$ is the length of the structure in the $y$ direction. The energy is mainly localized within the tube of width $L_{y}$ and thickness $(2 \kappa)^{-1}$ determined by the $e$ times intensity decrease. Such an evanescent wave is scattered by the nanospheres and excites the electric-dipole moments. They radiate and, thus, create a propagating wave in the 

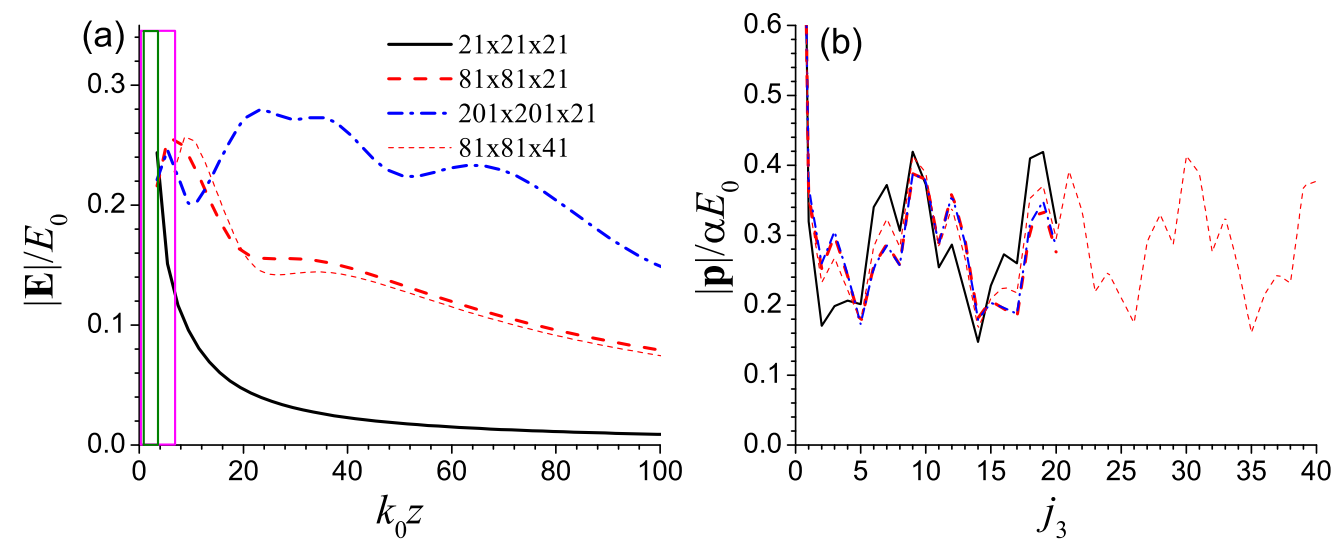

FIG. 3. Propagation of the coherently exiting evanescent wave $\left(k_{x}=2 \pi / R\right)$ depending on the geometrical size of the slab $\left(N_{x}+1\right) \times$ $\left(N_{y}+1\right) \times\left(N_{z}+1\right)\left(1 \mathrm{D}\right.$ direct calculations). (a) Dimensionless electric field $|\mathbf{E}| / E_{0}$ vs $k_{0} z$ and (b) dipole moment $|\mathbf{p}| / \alpha E_{0}$ vs position of dipole $j_{3}$ at $j_{1}=j_{2}=0$. Smaller and larger rectangles correspond to the slabs of 21- and 41-nanosphere thickness, respectively.

$z$ direction. We would like to estimate the share of energy that is redirected to the $z$ oriented tube $L_{y}$ by $(2 \kappa)^{-1}$. The redirected energy flux reads

$$
\Phi_{z}=\int_{0}^{(2 \kappa)^{-1}} \frac{c L_{y}}{8 \pi} \operatorname{Re}\left(\mathbf{E} \times \mathbf{H}^{*}\right)_{z} d x .
$$

The ratio $\eta=\Phi_{z} / \Phi_{x}^{e v}$ characterizes the efficiency of the conversion. In the case of the coherent excitation of dipoles $\eta$ is about $10^{-3}$, otherwise it is much less; e.g., $\eta \sim 10^{-6}$ for $k_{x}=10 k_{0}$. In the absence of the intrinsic structure (for continuous media) the evanescent waves are not scattered, and the mechanism of the conversion described above is not valid. It should be emphasized that, even in the best case of coherent excitation, the efficiency of conversion and transmission is still very low. It may not be sufficient for improving the lens resolution.

In the case of coherent excitation, the transmitted electric field depends on the size of the slab as shown in Fig. 3(a). Here we employ 1D direct calculations, because the coherent incident wave ensures the same field at the centers of all nanoparticles in the assembly. When the number of nanospheres is small $\left(N_{x}=N_{y}=20\right)$, the near field disappears quite rapidly.
However, the larger slab's dimension enhances the transmitted field. Similar result was reported for any evanescent wave in Ref. [26], but here we have the enhancement only for the waves in the band Eq. (6). When we double the thickness of the slab (dashed curve for $N_{z}=40$ ), the transmission weakly changes; that is, the transverse size of the slab determined by $N_{x}$ and $N_{y}$ is more important for propagation due to the coherent excitation. The dipole moments shown in Fig. 3(b) behave similarly for every slab's size. This means that increasing the transverse size we just accumulate more radiation.

The transmitted electric field changes when the nanosphere polarizability $\alpha=R^{3}\left(n^{2}-1\right) /\left(n^{2}+2\right)$ alters. It can be realized with the help of sphere's radius $R$ or refractive index $n$. Effect of the radius is stronger because of the $R^{3}$ dependence. Figure 4(a) indeed demonstrates a noticeable enhancement of the output in the regime of coherent excitation, while the influence of the refractive index is humble. For bigger spheres $(R=15 \mathrm{~nm})$, the oscillations of the dipole moment are more pronounced and the period of the oscillations is twice smaller compared with $R=7.5 \mathrm{~nm}$. Although the larger radii are beneficial for the transmission, one need to take care of validity of the electric-dipole approximation.
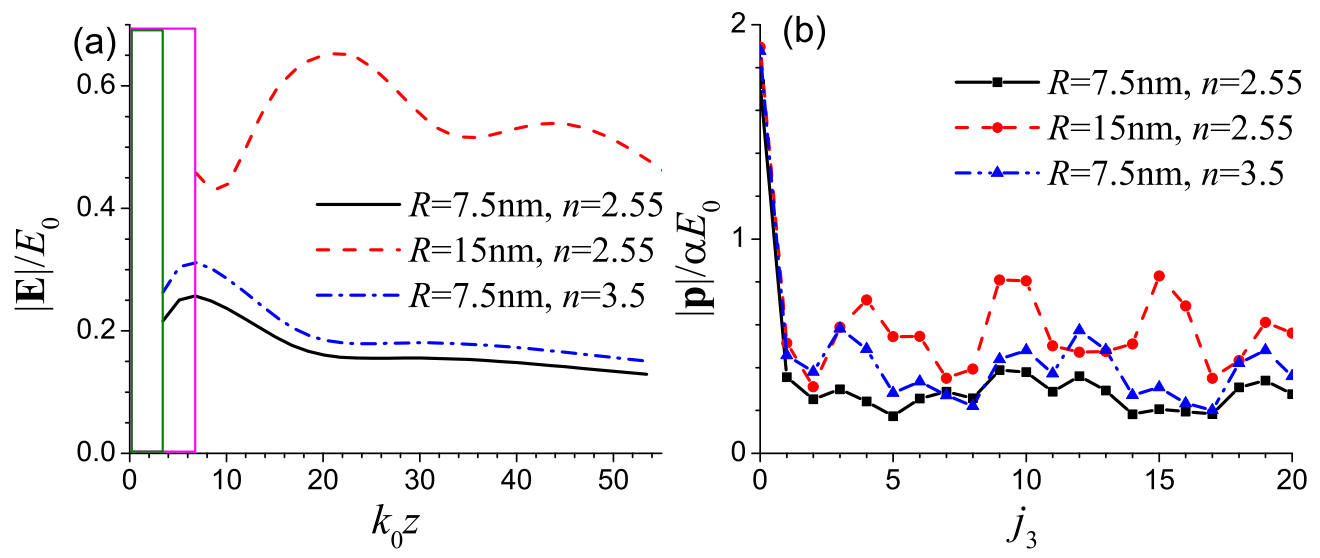

FIG. 4. Propagation of the coherently exiting evanescent wave $\left(k_{x}=2 \pi / R\right)$ depending on the parameters of the nanospheres (1D direct calculations). (a) Dimensionless electric field $|\mathbf{E}| / E_{0}$ vs $k_{0} z$ and (b) dipole moment $|\mathbf{p}| / \alpha E_{0}$ vs position of dipole $j_{3}$ at $j_{1}=j_{2}=0$. Smaller and larger rectangles correspond to the slabs composed of 7.5-nm- and 15-nm-radius nanospheres. The dimensions of the slab are $N_{x}=N_{y}=80$ and $N_{z}=20$. 


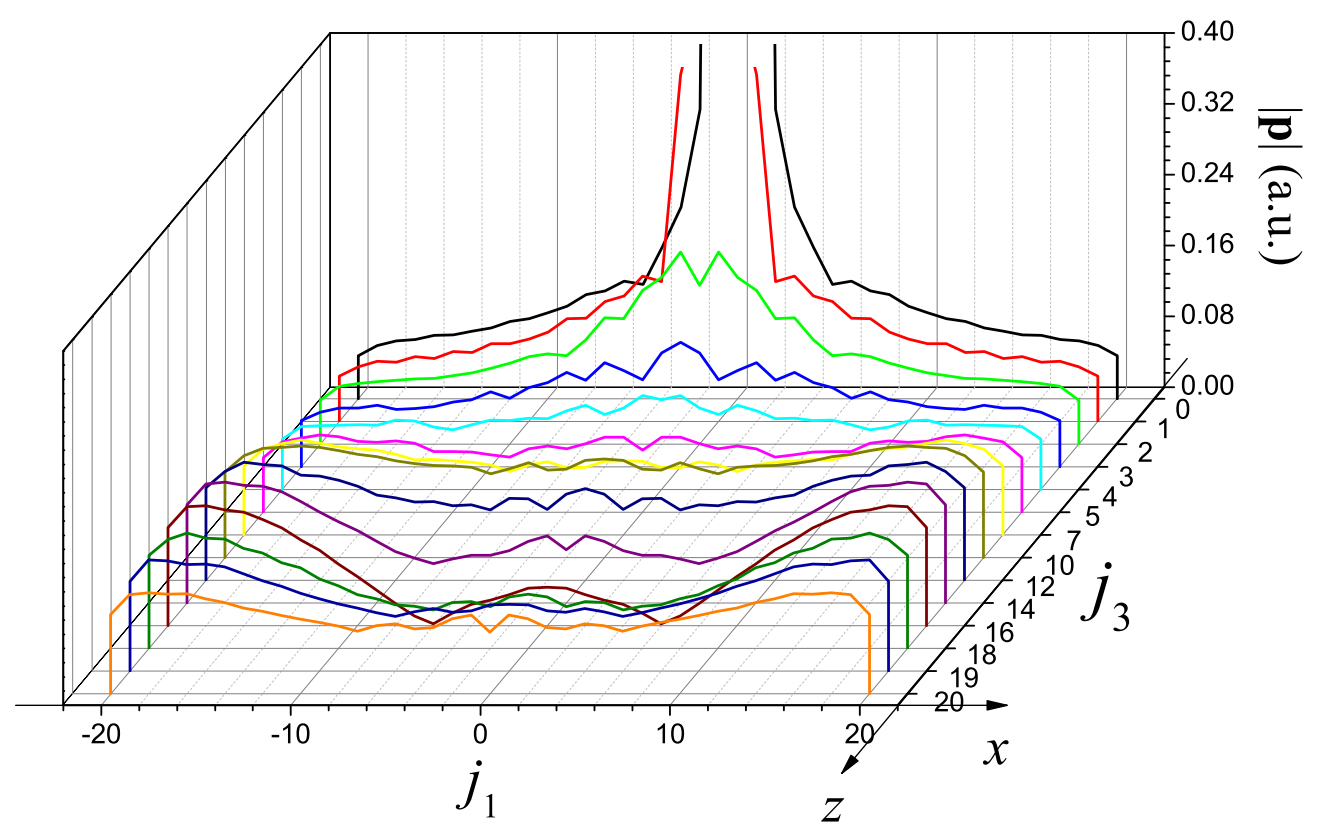

FIG. 5. 2D map of the dipole moment $|\mathbf{p}|$ distribution in the slab, when the point source is placed at $z_{0}=-1 \mathrm{~nm}$ and $x=y=0$. The size of the slab is $N_{x}=40, N_{y}=80$, and $N_{z}=20$.

\section{SCATTERING OF POINT SOURCE RADIATION}

Now we will consider features of the point-source-field propagation in the slab of nanospheres noticed in Ref. [26]. To perform 2D calculations we need an in-plane point source that can be presented as a thread of infinite length along the $y$ axis. The thread emits a $2 \mathrm{D}$ electric field $\mathbf{E}_{P S}\left(\mathbf{u}_{1}, \mathbf{u}_{2}\right)=$ $\hat{G}_{2 \mathrm{D}}\left(\mathbf{u}_{1}, \mathbf{u}_{2}\right) \mathbf{p}_{s}$, where $\mathbf{u}=(x, 0, z)$ is the $2 \mathrm{D}$ radius vector, $\mathbf{p}_{s}=\mathbf{e}_{y}$ is the dipole moment of the thread, and

$$
\begin{aligned}
\hat{G}_{2 \mathrm{D}}\left(\mathbf{u}_{1}-\mathbf{u}_{2}\right)= & i \pi H_{1}^{(1) \prime}\left(k_{0}\left|\mathbf{u}_{1}-\mathbf{u}_{2}\right|\right) \\
& \times\left(1-\frac{\left(\mathbf{u}_{1}-\mathbf{u}_{2}\right) \otimes\left(\mathbf{u}_{1}-\mathbf{u}_{2}\right)}{\left(\mathbf{u}_{1}-\mathbf{u}_{2}\right)^{2}}\right)
\end{aligned}
$$

is the electric Green's function. In Eq. (9), $H_{1}^{(1) \prime}$ is the derivative of the Hankel function of the first kind.
When the source is placed close to the slab of nanospheres [see Fig. 1(b)], it induces electric-dipole moments, as shown in Fig. 5. The nanospheres close to the source are strongly coupled to its radiation and one can observe the high peak in Fig. 5 near $z=0$. However, this peak promptly disappears approaching another slab boundary. Distribution of the dipole moments over $j_{3}$ at $j_{1}=0$ is fairly similar to the oscillating distribution, e.g., in Fig. 3(b).

According to Ref. [26], two point sources at subwavelength distance $d$ can be distinguished behind the slab of nanoparticles. To elucidate this assertion we perform similar calculations for the superposition of fields of two point sources $\mathbf{E}_{\text {inc }}=\mathbf{E}_{P S}\left(\mathbf{u}-\mathbf{u}_{+}\right)+\mathbf{E}_{P S}\left(\mathbf{u}-\mathbf{u}_{-}\right)$, where $\mathbf{u}_{ \pm}=$ $\left( \pm d / 2,0, z_{0}\right)$. Distribution of electric-dipole moments in Fig. 6(a) clearly demonstrates the propagating character of the field inside the composite slab. However, the transverse
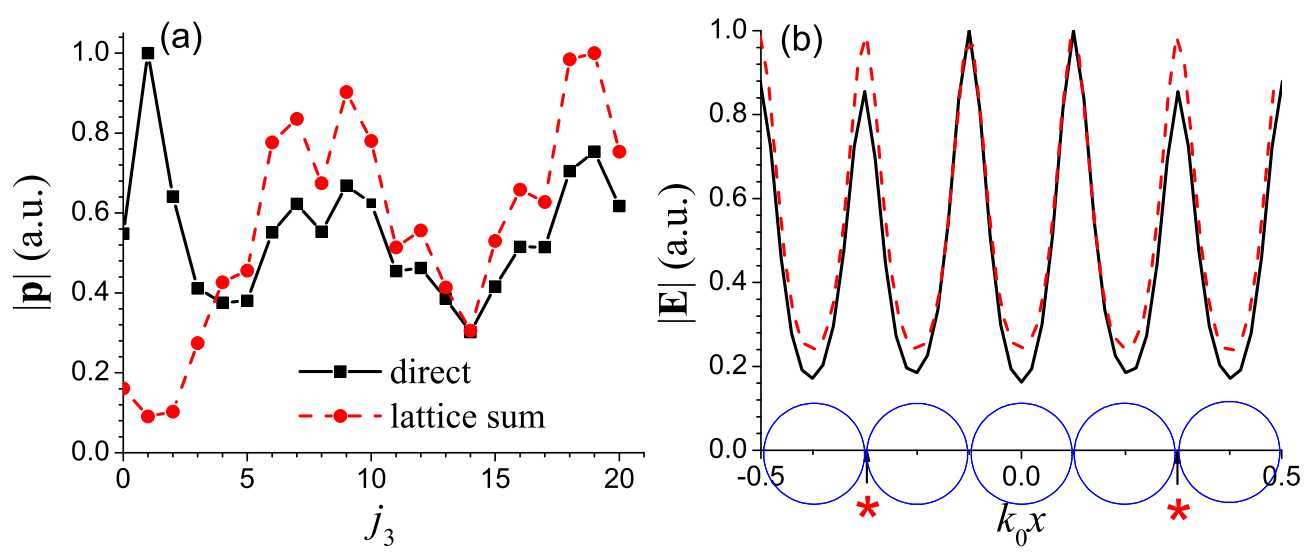

FIG. 6. Radiation propagation through the slab of nanospheres for two point sources at the distance $d=6 R$. Distribution of (a) the dipole moment $|\mathbf{p}|$ vs $j_{3}$ for $j_{1}=j_{2}=0$ and (b) electric field $|\mathbf{E}|$ vs $k_{0} x$ at $z=2 \sqrt{2 / 3} R N_{z}$. Normalization in plots guarantees the values in the range from 0 to 1 . Point sources marked by the arrows are placed at $x= \pm d / 2$ and $z_{0}=-1 \mathrm{~nm}$. Size of the slab: $N_{x}=N_{y}=N_{z}=20$ for lattice sum and $N_{x}=N_{z}=20, N_{y}=80$ for $2 \mathrm{D}$ direct calculations. 

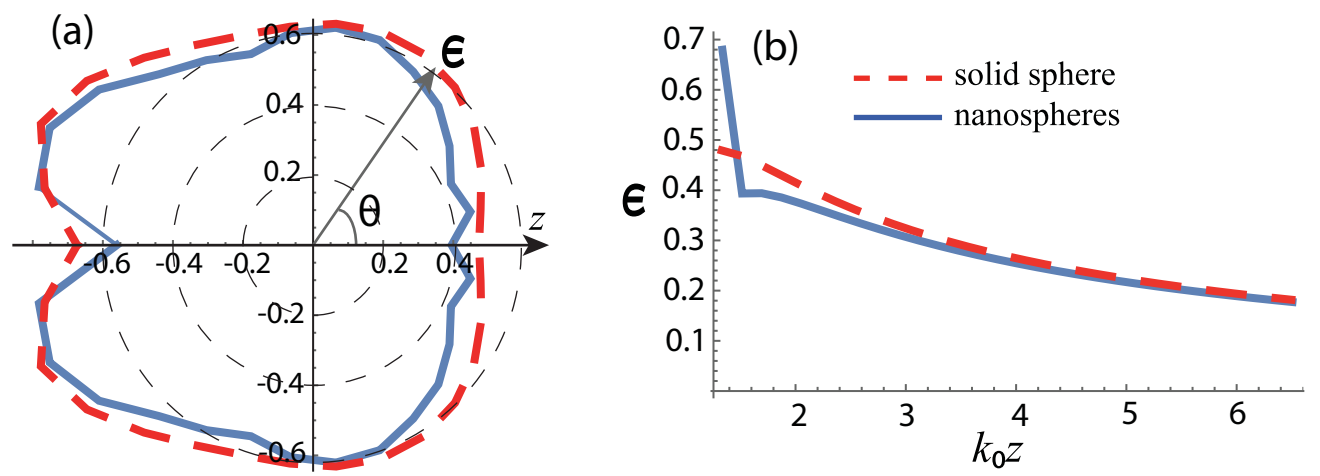

FIG. 7. Comparison of the field distributions $|\mathbf{E}(r, \theta, \varphi)|$ scattered by the solid sphere of radius $R_{0}=100 \mathrm{~nm}$ and spherical cluster of nanospheres of the same radius. (a) Angular distributions of the dimensionless scattered field $\epsilon\left(1.1 R_{0}, \theta, 0\right)=\left|\mathbf{E}\left(1.1 R_{0}, \theta, 0\right)\right| / k_{0}^{3} p_{0}$. (b) Longitudinal distributions of the field $\epsilon(z, 0,0)$. Position of the point source $\mathbf{p}=p_{0} \mathbf{e}_{x}$ is $z=-2.1 R_{0}$. Effective refractive index of the solid sphere $n_{\text {eff }}=2$ and wavelength $\lambda=470 \mathrm{~nm}$.

$x$ distribution of the transmitted electric field in Fig. 6(b) does not provide any evidence of two sources positioned at $k_{0} x \approx \pm 0.3$. In the near field we inspect the last row of the nanospheres in Fig. 6(b). In the far field we see a nonstructured output field (not shown). The far field has a Gaussian-like profile due to the finiteness of the structure in direct calculations. Thus, the fields of the point sources are intermixed by the cluster of nanospheres and we do not observe the subwavelength features. We think that hardly a very wide structure (large $N_{x}$ and $N_{y}$ ) as in Ref. [26] would improve the resolution, because the localization of the point sources makes the remote parts of the slab much less involved; see the transverse spread of the dipole moments in Fig. 5.

\section{SOLID SPHERE VS ASSEMBLY OF NANOSPHERES}

Albeit a far-field superresolution has not been confirmed for planar slabs of nanoparticles, the question of how the spherical shape affects the resolution is still open. To shed light on this situation we now look for any differences in the far-field scattering of two objects: a composite sphere of radius $R_{0}$ composed of nanospheres and a solid particle of the same radius $R_{0}$ and effective refractive index $n_{\text {eff }}$ obtained after homogenization of the nanospheres assembly. Wave propagation in the densely packed assembly can be calculated by using the direct three-dimensional (3D) technique as described in Sec. II. Coordinates of nanosphere centers are limited by the radius $R_{0}$ according to (see Appendix E for details)

$$
x^{2}+y^{2}+z^{2} \leqslant R_{0}^{2} .
$$

As an example, we compare two objects: a solid sphere of radius $R_{0}=100 \mathrm{~nm}$ and assembly of 672 nanospheres bounded by the same radius. We consider the same nanoparticles as before ( $R=7.5 \mathrm{~nm}, n=2.55)$. The refractive index of the solid sphere is the effective refractive index of the closely packed cluster $n_{\text {eff }}=2$. The incident field is generated by the point source with dipole moment $\mathbf{p}=p_{0} \mathbf{e}_{x}$ at $z=-2.1 R_{0}$, the coordinates of the center of the sphere or assembly being $x=y=z=0$. According to Fig. 7(a) the scattering pattern in the near field differs for the assembly of nanospheres and solid sphere. The difference is most pronounced for the forward $\left(\theta=0^{\circ}\right)$ and backward $\left(\theta=180^{\circ}\right)$ scattering. However, when the near fields die out, the far fields well agree with each other, as can be noticed in Fig. 7(b). We could get a better coincidence of the fields scattered by two spheres if the nanospheres were smaller. Anyway, we do not observe the influence of sphere's discrete structure on the far field. Therefore, the nanospheres in the mSIL are not expected to lead to the additional superresolution in the far field as it is claimed in Ref. [26]. The far-field behavior of the assembly of the nanospheres can be well explained by the high effective refractive index of the microsphere.

\section{CONCLUSION}

We have studied the mechanism of superresolution behind the concept of the metamaterial solid immersion lenses introduced in Refs. [26,27]. We exploit multiple-scattering theory, taking into account only electric-dipole moments of individual scatterers (dielectric nanospheres). In contrast to the results in Ref. [26], we have revealed a conversion of evanescent to propagating waves only in special conditions of the coherent excitation of the dipole moments in the cluster. Thus, some initial evanescent waves can indeed contribute to the far-field pattern, but efficiency of conversion is not high. To examine whether the conversion of evanescent to propagating waves is enough for improving the resolution, we calculated transmission of the radiation of two point sources separated by a subwavelength distance. We found a rapid diffraction of the radiation in the cluster that forbids observation of individual images of the point sources. It should be stressed that this result contradicts the guiding-like propagation and subwavelength imaging presented in Ref. [26]. Far-field scattering by a spherically shaped cluster of nanospheres is fairly similar to the scattering by a solid sphere of the same radius and effective refractive index. Therefore, we conclude that the internal nanostructure of the mSIL cannot explain its superresolving properties. We rather inclined to justify the improved resolution with the properties of conventional solid immersion lenses, for which the refractive index and magnification due to the spherical shape go hand in hand in order to increase the numerical aperture. 


\section{ACKNOWLEDGMENTS}

The authors would like to acknowledge financial support from the Villum Fonden, Denmark via the DarkSild project. A.N. is thankful for the financial support from the Belarusian Republican Foundation for Fundamental Research (Grant No. F18R-021).

\section{APPENDIX A: LATTICE DESCRIPTION}

Positions of nanospheres in the $\mathrm{AB}$ lattice shown in Fig. 1(a) can be presented by using the radius-vector

$$
\mathbf{r}_{i_{1}, i_{2}, i_{3}}=\sum_{k=1}^{3} i_{k} \mathbf{a}_{k},
$$

where $\mathbf{a}_{1}=2 R \mathbf{e}_{x}, \quad \mathbf{a}_{2}=R \mathbf{e}_{x}+\sqrt{3} R \mathbf{e}_{y}, \quad$ and $\quad \mathbf{a}_{3}=R \mathbf{e}_{x}+$ $\frac{R}{\sqrt{3}} \mathbf{e}_{y}+\frac{2 \sqrt{2} R}{\sqrt{3}} \mathbf{e}_{z}$ are the primitive lattice vectors. Integer numbers $i_{k}$ run over the spheres in the directions pointed out by the lattice vectors.

Position of a nanosphere in the assembly could be specified in another way. After substituting lattice vectors $\mathbf{a}_{k}$ the radius vector takes the form

$\mathbf{r}_{i_{1}, i_{2}, i_{3}}=\left(2 i_{1}+i_{2}+i_{3}\right) R \mathbf{e}_{x}+\left(3 i_{2}+i_{3}\right) \frac{R}{\sqrt{3}} \mathbf{e}_{y}+\frac{2 \sqrt{2} R}{\sqrt{3}} i_{3} \mathbf{e}_{z}$.

Then the $x$ and $z$ components of the radius vector can be described by independent integer indices $j_{1}=2 i_{1}+i_{2}+i_{3}$ and $j_{3}=i_{3}$, respectively. The position of the nanosphere along the $y$ axis depends on its $x$ and $z$ coordinates (indices $j_{1}$ and $\left.j_{3}\right)$ as $3 i_{2}+i_{3}=3 j_{1}-2 j_{3}-6 j_{2}$, where $j_{2}=i_{1}$. Denoting $\mathbf{b}_{1}=R \mathbf{e}_{x}, \mathbf{b}_{2}=\frac{R}{\sqrt{3}} \mathbf{e}_{y}$, and $\mathbf{b}_{3}=\frac{2 \sqrt{2} R}{\sqrt{3}} \mathbf{e}_{z}$, we arrive at

$$
\mathbf{r}_{j_{1}, j_{2}, j_{3}}=j_{1} \mathbf{b}_{1}+\left(3 j_{1}-2 j_{3}-6 j_{2}\right) \mathbf{b}_{2}+j_{3} \mathbf{b}_{3} .
$$

The integer numbers $j_{1}, j_{2}$, and $j_{3}$ define positions of the nanospheres in the lattice. For fixed $j_{1}$ and $j_{3}$ we have a number of spheres in the $y$ direction separated by the distance $6\left|\mathbf{b}_{2}\right|=2 \sqrt{3} R$ one from the other. Adding a multiple of six is equivalent to changing the index $j_{2}$ by 1 . Furthermore, we present the radius vector as

$$
\mathbf{r}_{j_{1}, j_{2}, j_{3}}=j_{1} \mathbf{b}_{1}+\left[\operatorname{Mod}\left(3 j_{1}-2 j_{3}, 6\right)-6 j_{2}\right] \mathbf{b}_{2}+j_{3} \mathbf{b}_{3},
$$

where $\operatorname{Mod}\left(3 j_{1}-2 j_{3}, 6\right)$ means the remainder of the division by six. Radius vector (A4) is better suited for the description of planar slabs composed of nanospheres.

\section{APPENDIX B: DIRECT CALCULATION}

Equation (2) can be solved directly without further assumptions to find the individual dipole moments $\mathbf{p}_{j_{1}, j_{2}, j_{3}}$ of the nanospheres in the nodes of the lattice. Introducing tensor

$$
A_{j_{1}, j_{2}, j_{3}, j_{1}^{\prime}, j_{2}^{\prime}, j_{3}^{\prime}}=\delta_{j_{1}, j_{1}^{\prime}} \delta_{j_{2}, j_{2}^{\prime}} \delta_{j_{3}, j_{3}^{\prime}}-\alpha \hat{G}_{E}\left(\mathbf{r}_{j_{1}^{\prime}, j_{2}^{\prime}, j_{3}^{\prime}}-\mathbf{r}_{j_{1}, j_{2}, j_{3}}\right),
$$

one can rewrite Eq. (2) in the following form:

$$
\sum_{j_{1}^{\prime}, j_{2}^{\prime}, j_{3}^{\prime}} A_{j_{1}, j_{2}, j_{3}, j_{1}^{\prime}, j_{2}^{\prime}, j_{3}^{\prime}} \mathbf{p}_{j_{1}^{\prime}, j_{2}^{\prime}, j_{3}^{\prime}}=\alpha \mathbf{E}_{\mathrm{inc}}\left(\mathbf{r}_{j_{1}, j_{2}, j_{3}}\right) \text {. }
$$

For convenience in numerical calculations, instead of three indices $j_{1}, j_{2}$, and $j_{3}$ we introduce a single index of dimension $3\left(N_{x}+1\right)\left(N_{y}+1\right)\left(N_{z}+1\right)$. Then Eq. (B2) can be represented in terms of multidimensional vectors $\vec{P}, \vec{E}$ and matrix $\mathcal{A}$ as

$$
\mathcal{A} \vec{P}=\alpha \vec{E} .
$$

The dipole moments of all nanospheres in the assembly are formally obtained by inversion of the square matrix $\mathcal{A}$ :

$$
\vec{P}=\alpha \mathcal{A}^{-1} \vec{E} .
$$

It should be stressed that this is an accurate technique for finding the response of a finite structure. The method described above is called the multiple-scattering technique [28-30]. The higher-order multipoles might be taken into account as well.

In general, the dimension of matrix $\mathcal{A}$ is large and, therefore, computations are challenging. To mitigate the computations we can assume that the incident field is two-dimensional (2D); that is, it depends only on two coordinates as $\mathbf{E}_{\text {inc }}(x, z)$. Examples of such fields are widely exploited and include obliquely incident and evanescent fields as well as fields of $2 \mathrm{D}$ point sources (a string along the $y$ axis). Then we assume that the electric-dipole moments of the spheres characterized by a couple of indices $j_{1}$ and $j_{3}$ are the same. Obviously, the thicker is the cluster in the $y$ direction, the better works the approximation. In this case, the electric-dipole moment reads

$$
\mathbf{p}_{j_{1}, j_{3}}=\alpha \sum_{j_{1}^{\prime}, j_{3}^{\prime}} A_{j_{1}, j_{3}, j_{1}^{\prime}, j_{3}}^{-1} \mathbf{E}_{\mathrm{inc}}\left(\mathbf{r}_{j_{1}^{\prime}, 0, j_{3}^{\prime}}\right)
$$

where

$$
A_{j_{1}, j_{3}, j_{1}^{\prime}, j_{3}^{\prime}}=\delta_{j_{1}, j_{1}^{\prime}} \delta_{j_{3}, j_{3}^{\prime}}-\sum_{j_{2}^{\prime}} \alpha \hat{G}_{E}\left(\mathbf{r}_{j_{1}^{\prime}, j_{2}^{\prime}, j_{3}^{\prime}}-\mathbf{r}_{j_{1}, 0, j_{3}}\right) .
$$

The lower dimension $3\left(N_{x}+1\right)\left(N_{z}+1\right)$ of the vectors and matrices allows us to reduce the time consumption of the calculations. However, as long as we have a finite number of nanospheres in the $y$ direction, the assumption of the same dipole moments for the fixed $\left(j_{1}, j_{3}\right)$ is an approximation.

In the case of a normally incident plane wave, the field is of the form $\mathbf{E}_{\text {inc }}(z)$, and the dipole moments of the nanospheres in the plane $z=$ const. can be assumed to be the same. For a finite structure it is again an approximation. The electricdipole moments distributed along the $z$ direction read

$$
\mathbf{p}_{j_{3}}=\alpha \sum_{j_{3}^{\prime}} A_{j_{3}, j_{3}^{\prime}}^{-1} \mathbf{E}_{\text {inc }}\left(\mathbf{r}_{0,0, j_{3}^{\prime}}\right),
$$

where the $3\left(N_{z}+1\right)$-dimensional matrix is introduced as

$$
A_{j_{3}, j_{3}^{\prime}}=\delta_{j_{3}, j_{3}^{\prime}}-\sum_{j_{1}^{\prime}, j_{2}^{\prime}} \alpha \hat{G}_{E}\left(\mathbf{r}_{j_{1}^{\prime}, j_{2}^{\prime}, j_{3}^{\prime}}-\mathbf{r}_{0,0, j_{3}}\right) .
$$

In this one-dimensional (1D) case, the calculations are the fastest in expense of the very limited choice of the incident fields. It is worth mentioning that although an obliquely incident plane wave makes the problem two dimensional, the 1D model is still able to predict correct results in the special 
conditions of coherent excitation of the nanospheres. The coherent excitation means that all nanospheres in the plane $z=$ const. are excited in phase as in the 1D case.

The transmitted electric field is equal to the superposition of the incident field and fields of the induced electric dipoles as

$$
\mathbf{E}_{t r}(\mathbf{r})=\mathbf{E}_{\mathrm{inc}}(\mathbf{r})+\sum_{j_{1}, j_{2}, j_{3}} \hat{G}_{E}\left(\mathbf{r}-\mathbf{r}_{j_{1}, j_{2}, j_{3}}\right) \mathbf{p}_{j_{1}, j_{2}, j_{3}} .
$$

When this equation is applied in the reduced 2D and 1D cases, the dipole moments have to be $\mathbf{p}_{j_{1}, j_{3}}$ and $\mathbf{p}_{j_{3}}$, respectively.

\section{APPENDIX C: LATTICE-SUM CALCULATIONS}

Another simplifying approach for evaluation of scattered fields in periodic systems is with employment of lattice sums. It is based upon the idea that, due to the Floquet-Bloch theorem in periodic structures, it is possible to calculate the fields (electric-dipole moments) at any nanoparticle center using the field (dipole moment) at the center of one selected nanoparticle. Obviously, it is an accurate assumption for infinite structures and approximate for finite due to the edge effects. In the case of a thin film of nanoparticles, this assumption holds true for the nanospheres in the $(x, y)$ plane, but is violated along the $z$ axis. When a translationsymmetric incident wave (oblique plane wave) propagates in the $(x, z)$ plane, we can write the electric fields and dipole moments at the centers of nanospheres as, respectively, $\quad \mathbf{E}_{\mathrm{inc}}\left(\mathbf{r}_{j_{1}, j_{2}, j_{3}}\right)=\mathbf{E}_{\mathrm{inc}}\left(\mathbf{r}_{0,0, j_{3}}\right) \exp \left[i k_{x}\left(x_{j_{1}, j_{2}, j_{3}}-x_{0,0, j_{3}}\right)\right]$ and $\mathbf{p}_{j_{1}, j_{2}, j_{3}}=\mathbf{p}_{0,0, j_{3}} \exp \left[i k_{x}\left(x_{j_{1}, j_{2}, j_{3}}-x_{0,0, j_{3}}\right)\right]$. Then Eq. (2) for $j_{1}=j_{2}=0$ takes the form

$$
\sum_{j_{3}^{\prime}} A_{j_{3}, j_{3}^{\prime}} \mathbf{p}_{0,0, j_{3}^{\prime}}=\alpha \mathbf{E}_{\mathrm{inc}}\left(\mathbf{r}_{0,0, j_{3}}\right)
$$

where

$$
A_{j_{3}, j_{3}^{\prime}}=\delta_{j_{3}, j_{3}^{\prime}}-\alpha \sum_{j_{1}^{\prime}, j_{2}^{\prime}} \hat{G}_{E}\left(\mathbf{r}_{j_{1}^{\prime}, j_{2}^{\prime}, j_{3}^{\prime}}-\mathbf{r}_{0,0, j_{3}}\right) e^{i k_{x}\left(x_{\left.j_{1}^{\prime}, j_{2}^{\prime}, j_{3}^{\prime}-x_{0,0, j_{3}^{\prime}}\right)}\right.}
$$

Although Eq. (C1) has solutions as in the 1D case (B7), it is applicable for $2 \mathrm{D}$ obliquely incident plane waves now. In contrast to the 1D theory, the dipole moments are not equal, but rather governed by the Floquet-Bloch theorem. Finding $\left(N_{z}+1\right)$ values of $\mathbf{p}_{0,0, j_{3}}$, we obtain transmitted fields for the incident plane wave as

$$
\begin{aligned}
\mathbf{E}_{t r}(\mathbf{r})= & \mathbf{E}_{\mathrm{inc}}(0) e^{i k_{x} x+i \sqrt{k_{0}^{2}-k_{x}^{2}} z}+\sum_{j_{1}, j_{2}, j_{3}} \hat{G}_{E}\left(\mathbf{r}-\mathbf{r}_{j_{1}, j_{2}, j_{3}}\right) \\
& \times \mathbf{p}_{0,0, j_{3}} e^{i k_{x}\left(x_{j_{1}, j_{2}, j_{3}}-x_{0,0, j_{3}}\right)}
\end{aligned}
$$

In the case of arbitrary incident $2 \mathrm{D}$ field $\mathbf{E}_{\mathrm{inc}}(x, z)$, this theory should be applied for each Fourier component of the field, bringing us to

$$
\begin{aligned}
\mathbf{E}_{t r}(\mathbf{r})= & \mathbf{E}_{\mathrm{inc}}(x, z)+\sum_{j_{1}, j_{2}, j_{3}} \hat{G}_{E}\left(\mathbf{r}-\mathbf{r}_{j_{1}, j_{2}, j_{3}}\right) \\
& \times \mathbf{p}_{0,0, j_{3}}\left(x_{j_{1}, j_{2}, j_{3}}-x_{0,0, j_{3}}\right),
\end{aligned}
$$

where a spatial distribution of the dipole moment is determined by the Fourier transform as

$$
\mathbf{p}_{0,0, j_{3}}(x)=\int \mathbf{p}_{0,0, j_{3}}\left(k_{x}\right) \exp \left(i k_{x} x\right) d k_{x} .
$$

\section{APPENDIX D: COMPARISON OF DIRECT AND LATTICE-SUM TECHNIQUES}

An incident field appropriate for description of both propagating and evanescent waves reads

$$
\mathbf{E}_{\text {inc }}=E_{0} \exp \left(i k_{x} x+i \sqrt{k_{0}^{2}-k_{x}^{2}} z\right) \mathbf{e}_{y},
$$

where $E_{0}$ is the field amplitude. If $k_{x}<k_{0}\left(k_{x}>k_{0}\right)$, then the wave is propagating (evanescent). A normally incident plane wave has $k_{x}=0$. In the approximation $R \ll \lambda$, the nanospheres can be treated as electric dipoles with polarizability $\alpha=R^{3}\left(n^{2}-1\right) /\left(n^{2}+2\right)$.

The incident electric field Eq. (D1) corresponds to the wave in the plane $(x, z)$. Hence, it is justified to use the 2D model for direct calculations. The field outside the slab decreases as the distance from the slab grows; see Fig. 8(a), converging to the transmission coefficient of a homogenized layer of refractive index $n_{\text {eff }}=2$ [see the dashed horizontal line in Fig. 8(a)]. Such a far-field tail for the slab of nanoparticles has the same nature as that for the single electric dipole. The lattice-sum technique results in the similar curve, although the far-field limit is slightly overestimated. $N_{y}$ is taken as 80 for the direct calculations to ensure the validity of the 2D model ( $N_{y}$ should be as large as possible to avoid edge effects)

The transverse distribution of the transmitted field shows the peaks between the nanospheres, the peak value being the maximal near the center of the structure [Fig. 8(b)]. This distribution right behind the assembly of nanospheres is created by the near fields. Moving away from the cluster of nanospheres, the near fields (peaks) fade away keeping only the far-field transmission as for the continuous slab. In the lattice-sum calculations, we assume the periodicity, missing the edge effects; see the red line in Fig. 8(b).

Distribution of the nanosphere dipole moments for $j_{1}=$ $j_{2}=0$ behaves similarly within the framework of both calculation methods, as demonstrated in Fig. 8(c). Oscillations observed in Fig. 8(c) are a fingerprint of a propagating wave in the cluster of nanospheres. These oscillations have a wavelength about $150 \mathrm{~nm}$ and can be understood as standing waves in the slab of nanospheres. Transmission of an obliquely incident plane wave is determined by the more complicated interference picture; however, the oscillatory dependence of the dipole moments is preserved.

For the normally incident plane waves we could apply the $1 \mathrm{D}$ direct calculations. In this case, we do not take into account the edge effects of the finite structure in the sense that all nanospheres for the certain $j_{3}$ possess the same electricdipole moment. The value of the output field depends on the size of the nanosphere assembly. The larger numbers $N_{x}$ and 

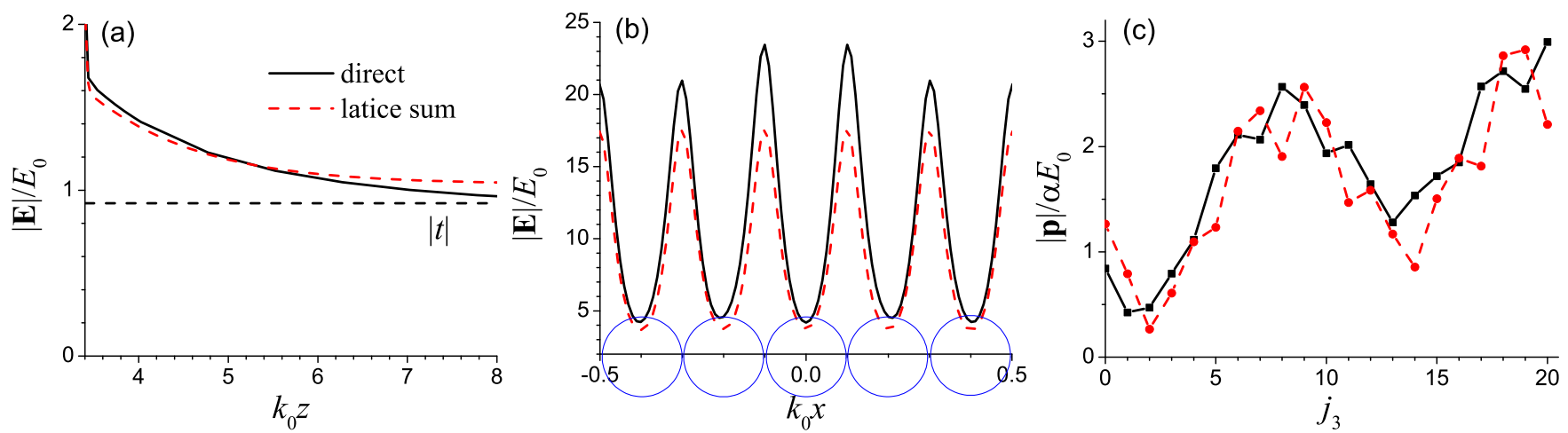

FIG. 8. Comparison of the $2 \mathrm{D}$ direct and lattice-sum calculations. (a) Electric field $|\mathbf{E}| / E_{0}$ of the wavelength $\lambda=470 \mathrm{~nm}$ transmitted by the slab of nanospheres (radius $R=7.5 \mathrm{~nm}$ and refractive index $n=2.55$ ) as a function of $k_{0} z\left(x=y=0\right.$ or $\left.j_{1}=j_{2}=0\right)$. (b) Transmission coefficient $|\mathbf{E}| / E_{0}$ as a function of coordinate $x$ at $z=2 \sqrt{2 / 3} R N_{z}$ corresponding to the center of the last sphere in the slab (circles demonstrate positions of the nanospheres). (c) Dimensionless electric-dipole moments $|\mathbf{p}| / \alpha E_{0}$ of the nanospheres vs $j_{3}$ (coordinate $z$ ) at $j_{1}=j_{2}=0$. Geometrical parameters of the slab: $N_{x}=20, N_{y}=80$, and $N_{z}=20$ for direct calculations and $N_{x}=N_{y}=N_{z}=20$ for lattice-sum calculations.

$N_{y}$ bring us closer to the fulfillment of the conditions of equal dipole moments in the plane.

\section{APPENDIX E: LIMITATIONS ON INDICES $j_{1}, j_{2}$, AND $j_{3}$ FOR A CLOSELY PACKED SPHERE}

Here we calculate the light scattering on the cluster of nanospheres having the shape of a sphere of radius $R_{0}$. Let us write the limitations on the integer numbers $j_{1}, j_{2}$, and $j_{3}$ defined in Eq. (A4) caused by the spherical shape. Number $j_{3}$ specifies the coordinate $-R_{0} \leqslant z \leqslant R_{0}$ for different cuts of the sphere. The discrete cuts are separated by the distance $2 \sqrt{2 / 3} R$; therefore,

$$
-\left[\frac{\sqrt{3} R_{0}}{2 \sqrt{2} R}\right] \leqslant j_{3} \leqslant\left[\frac{\sqrt{3} R_{0}}{2 \sqrt{2} R}\right]
$$

where $[x]$ is the integer part of $x$.
Within the cut determined by $j_{3}$ (for the fixed $z=$ $\left.2 \sqrt{2 / 3} R j_{3}\right)$, one writes $x^{2}+y^{2} \leqslant R_{0}^{2}-z^{2}=R_{0}^{2}-8 R^{2} j_{3}^{2} / 3$. The cuts along the $x$ axis occur at the equal distances $R$, hence,

$$
-\left[\frac{\sqrt{R_{0}^{2}-8 R^{2} j_{3}^{2} / 3}}{R}\right] \leqslant j_{1} \leqslant\left[\frac{\sqrt{R_{0}^{2}-8 R^{2} j_{3}^{2} / 3}}{R}\right] .
$$

Finally, we derive the bounds for integer number $j_{2}$ in the $y$ direction depending on $j_{1}$ and $j_{3}$ as

$$
a_{-} \leqslant j_{2} \leqslant a_{+},
$$

where

$$
a_{ \pm}=\left[-\frac{j_{1}}{2}+\frac{j_{3}}{3} \pm \frac{\sqrt{3 R_{0}^{2}-8 R^{2} j_{3}^{2}-3 R^{2} j_{1}^{2}}}{6 R}\right] .
$$

Such a cluster of nanospheres can be characterized by using the direct calculation technique based on the multiplescattering theory.
[1] J. B. Pendry, Negative Refraction Makes a Perfect Lens, Phys. Rev. Lett. 85, 3966 (2000).

[2] D. R. Smith and D. Schurig, Electromagnetic Wave Propagation in Media With Indefinite Permittivity and Permeability Tensors, Phys. Rev. Lett. 90, 077405 (2003).

[3] A. Poddubny, I. Iorsh, P. Belov, and Y. Kivshar, Hyperbolic metamaterials, Nat. Photonics 7, 948 (2013).

[4] Z. Jacob, L. V. Alekseyev, and E. Narimanov, Optical hyperlens: Far-field imaging beyond the diffraction limit, Opt. Express 14, 8247 (2006).

[5] Z. Liu, H. Lee, Y. Xiong, C. Sun, and X. Zhang, Far-field optical hyperlens magnifying sub-diffraction-limited objects, Science 315, 1686 (2007).

[6] I. I. Smolyaninov, Y.-J. Hung, and C. C. Davis, Magnifying superlens in the visible frequency range, Science 315, 1699 (2007).

[7] T. Repän, A. V. Lavrinenko, and S. V. Zhukovsky, Dark-field hyperlens: Super-resolution imaging of weakly scattering objects, Opt. Express 23, 25350 (2015).
[8] A. Novitsky, T. Repän, S. V. Zhukovsky, and A. V. Lavrinenko, Subwavelength hyperlens resolution with perfect contrast function, Ann. Phys. (Berlin, Ger.) 530, 1700300 (2018).

[9] Z. Wei, Y. Cao, Z. Gong, X. Su, Y. Fan, C. Wu, J. Zhang, and H. $\mathrm{Li}$, Subwavelength imaging with a fishnet flat lens, Phys. Rev. B 88, 195123 (2013).

[10] D. Kitayama, M. Yaita, and H.-J. Song, Laminated metamaterial flat lens at millimeter-wave frequencies, Opt. Express 23, 23348 (2015).

[11] P. R. West, J. L. Stewart, A. V. Kildishev, V. M. Shalaev, V. V. Shkunov, F. Strohkendl, Y. A. Zakharenkov, R. K. Dodds, and R. Byren, All-dielectric subwavelength metasurface focusing lens, Opt. Express 22, 26212 (2014).

[12] B. Xu, Z. Wei, C. Wu, Y. Fan, Z. Wang, and H. Li, Neardiffraction-limited focusing with gradient high-impedance metasurface, Opt. Mater. Express 7, 1141 (2017).

[13] S. Colburn, A. Zhan, and A. Majumdar, Metasurface optics for full-color computational imaging, Sci. Adv. 4, eaar2114 (2018). 
[14] J. C. Ginn, I. Brener, D. W. Peters, J. R. Wendt, J. O. Stevens, P. F. Hines, L. I. Basilio, L. K. Warne, J. F. Ihlefeld, P. G. Clem, and M. B. Sinclair, Realizing Optical Magnetism from Dielectric Metamaterials, Phys. Rev. Lett. 108, 097402 (2012).

[15] D. Bao, K. Z. Rajab, Y. Hao, E. Kallos, W. Tang, C. Argyropoulos, Y. Piao, and S. Yang, All-dielectric invisibility cloaks made of $\mathrm{BaTiO}_{3}$-loaded polyurethane foam, New J. Phys. 13, 103023 (2011).

[16] Z. Hayran, H. Kurt, R. Herrero, M. Botey, and K. Staliunas, All-dielectric self-cloaked structures, ACS Photonics 5, 2068 (2018).

[17] S. Jahani, S. Kim, J. Atkinson, J. C. Wirth, F. Kalhor, W. D. Newman, P. Shekhar, K. Han, V. Van, R. G. DeCorby, L. Chrostowski, M. Qi, and Z. Jacob, Controlling evanescent waves using silicon photonic all-dielectric metamaterials for dense integration, Nat. Commun. 9, 1893 (2018).

[18] D. G. Baranov, D. A. Zuev, S. I. Lepeshov, O. V. Kotov, A. E. Krasnok, A. B. Evlyukhin, and B. N. Chichkov, All-dielectric nanophotonics: The quest for better materials and fabrication techniques, Optica 4, 814 (2017).

[19] Yu. Kivshar, All-dielectric meta-optics and non-linear nanophotonics, Natl. Sci. Rev. 5, 144 (2018).

[20] S. Jahani and Z. Jacob, All-dielectric metamaterials, Nat. Nanotechnol. 11, 23 (2016).

[21] S. M. Mansfield and G. S. Kino, Solid immersion microscope, Appl. Phys. Lett. 57, 2615 (1990).
[22] D. A. Fletcher, K. B. Crozier, K. W. Guarini, S. C. Minne, G. S. Kino, C. F. Quate, and K. E. Goodson, Microfabricated silicon solid immersion lens, J. Microelectromech. Syst. 10, 450 (2001).

[23] M.-S. Kim, T. Scharf, M. T. Haq, W. Nakagawa, and H. P. Herzig, Subwavelength-size solid immersion lens, Opt. Lett. 36, 3930 (2011).

[24] E. Abbe, On new methods for improving spherical correction applied to the construction of wide-angled object-glasses, J. R. Microsc. Soc. 2, 812 (1879).

[25] L. Novotny and B. Hecht, Principles of Nano-Optics (Cambridge University Press, Cambridge, 2012).

[26] W. Fan, B. Yan, Z. Wang, and L. Wu, Three-dimensional alldielectric metamaterial solid immersion lens for subwavelength imaging at visible frequencies, Sci. Adv. 2, e1600901 (2016).

[27] H. Zhu, W. Fan, S. Zhou, M. Chen, and L. Wu, Polymer colloidal sphere-based hybrid solid immersion lens for optical super-resolution imaging, ACS Nano 10, 9755 (2016).

[28] L. L. Foldy, The multiple scattering of waves. I. General theory of isotropic scattering by randomly distributed scatterers, Phys. Rev. 67, 107 (1945).

[29] M. Lax, Multiple scattering of waves, Rev. Mod. Phys. 23, 287 (1951).

[30] X. Wang, X.-G. Zhang, Q. Yu, and B. N. Harmon, Multiplescattering theory for electromagnetic waves, Phys. Rev. B 47, 4161 (1993). 